\title{
The Meyer-Neldel Rule in Semiconductors
}

\author{
R. METSELAAR AND G. OVERSLUIZEN* \\ Laboratory of Physical Chemistry, Eindhoven University of Technology, \\ Eindhoven, The Netherlands
}

Received January 31, 1984; in revised form June 5, 1984

\begin{abstract}
Two explanations are given for the Meyer-Neldel rule in inorganic semiconductors. First it is shown that the freezing-in of donor acceptor-type defects can lead to this rule both for band conductors and for small-polaron hopping conductors. Next it is shown that a Gaussian distribution of defect energy levels or a Gaussian distribution of hopping energies, under specific conditions of defect interactions, can also lead to this rule. As an example of a small-polaron hopping conductor experimental results are described of conductivity measurements on a vanadium garnet single crystal. The first-mentioned model leads to a freezing-in temperature which corresponds well with the value known from other experiments. $\mathcal{1} 1984$ Academic Press, Inc.
\end{abstract}

\section{Introduction}

The temperature dependence of the electrical conductivity of solids is given by the formula

$$
\sigma=A \exp \left(-E_{\mathrm{a}} / k T\right) .
$$

This equation is valid for broad-band conductors. In the case of small-polaron hopping conductors or ionic conducting solids one has to use

$$
\sigma T=A \exp \left(-E_{\mathrm{a}} / k T\right) .
$$

The term $A$ is called the preexponential factor. The use of this word is not quite justified, however, since $A$ often contains an exponential term itself. It was first pointed out in a series of articles by Meyer (1-4) that for the semiconducting oxides $\mathrm{UO}_{2}, \mathrm{Fe}_{2} \mathrm{O}_{3}$, $\mathrm{ZnO}$, and $\mathrm{TiO}_{2}$ a linear relationship holds of the form

* Present affiliation: Philips Research Laboratories, Eindhoven, The Netherlands.

0022-4596/84\$3.00

Copyright 1984 by Academic Press, Inc

All rights of reproduction in any form reserved.

$$
\ln A=\alpha E_{\mathrm{a}}+\beta .
$$

This relation is called the Meyer-Neldel rule.

The same phenomenon was found later for $\mathrm{BaTiO}_{3}$ and $\mathrm{SiC}(5)$ and for $\mathrm{Cu}_{2} \mathrm{O}(6)$. Figure 1 gives a survey of the results obtained by Busch. The measurements of Busch were obtained on polycrystalline samples, the measurements of Weichman and Kuzel on single crystals. The data points for a given compound were obtained on samples which were prepared or annealed under different conditions. It is seen from the figure that for each of these compounds the so-called Meyer-Neldel rule holds.

In 1967 Gutmann and Lyons (7) made a plot of $\log A$ versus $E_{\mathrm{a}}$ for a large group of organic semiconductors and found the data to roughly follow Eq. (3). Soon hereafter Rosenberg et al. (8) reported that Eq. (3) also applies for a single semiconducting organic compound where $E_{\mathrm{a}}$ is varied by hy320 


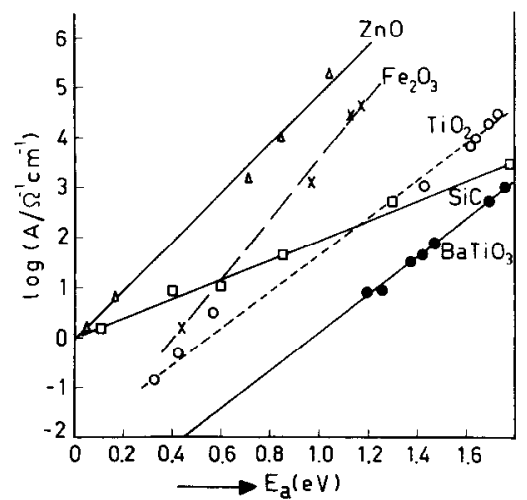

Fig. 1. Plot of the preexponential constant $A$ as a function of the activation energy of the conductivity. (Data from Ref. (5).)

dration and complex formation. Instead of Eq. (1) a three-constant equation holds

$$
\sigma=A^{\prime} \exp \left(E_{\mathrm{a}} / k T_{0}\right) \exp \left(-E_{\mathrm{a}} / k T\right) .
$$

In this equation a characteristic temperature $T_{0}$ is introduced. Though in organic semiconductors this relation is often called the compensation rule, it is of course equivalent to the Meyer-Neldel rule as given in Eq. (3), with $\left(k T_{0}\right)^{-1}=\alpha$ and $\ln A^{\prime}=\beta$. Equation (4) has also been used to describe conductivity measurements in amorphous organic semiconductors by Roberts and Thomas (9).

Since the measurements on $\mathrm{Cu}_{2} \mathrm{O}$ in 1969 no further examples of the Meyer-Neldel rule in inorganic semiconductors have been reported until 1977 . Since that time several authors mentioned that this rule also applies to hydrogenated amorphous silicon (a$\mathrm{Si}: \mathrm{H})$, both in undoped samples $(10)$, in doped samples (II) and in connection with light-induced conductivity changes (the Staebler-Wronski effect) (12-14).

Further, it was shown by Dosdale and Brook (15-17) that the rule is valid likewise for many data on diffusion or conductivity in ionic conductors. In that case Eq. (2) has to be used or the equivalent expression for the diffusion coefficient, $D=D_{0} \exp \left(-E_{\mathrm{a}} /\right.$ $k T)$.
In this paper we will present some new results obtained on single crystals of the garnet $\mathrm{Ca}_{2} \mathrm{NaMg}_{2} \mathrm{~V}_{3} \mathrm{O}_{12-x}$, where $x$ is varied by a high-temperature annealing treatment under varying oxygen pressures.

\section{Explanations for the Meyer-Neldel Rule}

\subsection{Inorganic Semiconductors}

The first explanation of the experimental data produced by Meyer was given by $\mathrm{Gi}-$ solf (18). However, it is fairly improbable that his explanation holds for the widely different compounds discussed by Meyer since the theory of Gisolf asks for very specific restrictions on the acceptor and donor levels and on the mobility of the charge carriers. A quite different theory was put forward by Busch (5). It is assumed that we are dealing with extrinsic, broad-band semiconductors where the donor concentration is frozen-in during cooling after the sintering procedure at high temperatures. According to Busch the constant $\alpha$ in Eq. (3) is related to the temperature $\theta$ where the donor concentration is frozen-in: $\alpha=1 /(2 k \theta)$. This equation is also used by Weichman and Kuzel (6) to explain the data obtained on a single crystal of $\mathrm{Cu}_{2} \mathrm{O}$.

Both for inorganic and organic semiconductors the Meyer-Neldel rule is observed in samples in which differences in concentration of charge carriers have been created by some chemical treatment. It can be expected quite generally that the activation energy will change when the defect concentration increases. Miller and Abrahams (19) made calculations for homopolar broadband semiconductors at low temperatures. At sufficiently high doping levels impurity conduction will play a role besides band conduction. Austin and Mott (20) applied this theory to polar materials. The activation cncrgy is found to decreasc proportionally to the concentration $x$ of the majority 
centers: $E_{\mathrm{a}}=E_{0}-C x^{1 / 3}$. Bosman and van Daal (21) use this expression to describe the decrease of $E_{\mathrm{a}}$ at $T<300 \mathrm{~K}$ for $p$-type $\mathrm{NiO}, \mathrm{CoO}$, and $\mathrm{MnO}$ and for $n$-type $\alpha$ $\mathrm{Fe}_{2} \mathrm{O}_{3}$. The fit of the experimental data with this equation is rather bad. Although impurity conduction does explain the decrease of $E_{\mathrm{a}}$ with increase of $x$ (increase of dope concentration), it does not lead to the Meyer-Neldel behavior.

In the case of a-Si : $\mathrm{H}$ no general explanation has been given so far.

\subsection{Organic Semiconductors}

The Meyer-Neldel rule has been observed in quite different crystalline organic compounds like cholesterol/donor (or /acceptor) complexes, hemoglobin in different hydration states, retinal complexes, nucleic acids $(8)$, or the amorphous solid violanthrene-iodine complexes. In all cases we are dealing with low mobility materials.

Kemeny and Rosenberg (22) derived an equation for the conductivity assuming electron tunneling through intermolecular barriers from the activated energy levels of the organic molecules. The characteristic temperature $T_{0}$ in Eq. (4) is than directly related to the height of the barrier. In a following publication these authors (23) show that also small-polaron band conduction can lead to Eq. (4), in which case $2 T_{0}$ equals the Debye temperature $T_{\mathrm{D}}$. This means however, that this theory is only applicable in the temperature region $T<T_{\mathrm{D}} / 4$.

Roberts (24) points out that a rectifying layer at the electrode-solid interface can also lead to the Meyer-Neldel rule. A second mechanism discussed by Roberts is possible if the concentration of the majority carrier band states tail exponentially with energy. This case could apply to amorphous solids (9).

\subsection{Ionic Conductors}

In ionic solids the decrease of the activation energy with increasing conductivity is also well known. Lidiard (25) has ascribed this effect to defect-defect interactions and applied the Debye-Hückel approach as developed for nonideal electrolyte solutions to ionic solids. Wapenaar et al. (26) were able to explain the Meyer-Neldel rule in $\mathrm{Ba}_{1-x} \mathrm{La}_{x} \mathrm{~F}_{2+x}$ solid solutions assuming a Gaussian distribution of activation energies where the average value depends linearly on the lanthanum concentration $x$. This behavior is found for concentrations $x>0.05$, where due to the defect interactions clusters are formed which can explain the broad distribution of activation energies. A more general treatment is given by Dosdale and Brook (15-17); these authors give three possible explanations for the occurrence of the Meyer-Neldel rule in ionic conductors. A trivial reason is that there are errors in $A$ and $E_{\mathrm{a}}$ due to wrong extrapolation of the $\log$ $\sigma$ versus $T^{-1}$ plots. One has to be aware of this danger, but there are too many reproducible results to accept this as a general explanation. A second possibility is that data are obtained in the temperature region where a transition from extrinsic to intrinsic behavior occurs. A third possibility is that there are two processes contributing simultaneously to $\sigma$ or $D$, the ratio of these two contributions being different in different samples. These arguments can be extended to semiconductors.

\section{Experimental Results for a Vanadium Garnet}

We have measured electrical transport properties of single crystals of the garnet $\mathrm{Ca}_{2} \mathrm{NaMg}_{2} \mathrm{~V}_{3} \mathrm{O}_{12-x}$ (27) grown from $\mathrm{PbO}$ and $\mathrm{V}_{2} \mathrm{O}_{5}$ fluxes. In the crystals as grown all vanadium ions are present as $\mathrm{V}^{5+}$ ions. After a reduction treatment at elevated temperatures in air or in $\mathrm{CO} / \mathrm{CO}_{2}$ gas mixtures, part of the vanadium is present as $\mathrm{V}^{4+}$. $\mathrm{By}$ quenching to room temperature crystals were obtained with different concentrations of $\mathrm{V}^{4+}$ in the $\mathrm{V}^{5+}$ sublattice. Results of the 
electrical conductivity measurements are shown in Fig. 2. The maximum measurement temperature was restricted to about $700 \mathrm{~K}$ to avoid slow reoxidation of the samples. No differences were observed between dc and ac measurements. The numbers in the figure correspond to samples which were annealed or quenched in different ways; details are given in Ref. (27). It can be seen from Fig. 2 that the curves 1-8 intersect at a temperature around 900-1000 $\mathrm{K}$. That these samples obey the MeyerNeldel rule can be seen more clearly from a plot of ${ }^{10} \log \sigma T$ versus $E_{\mathrm{a}}$ at a fixed temperature as shown in Fig. 3. The three curves drawn here for $T=400,500$ and $667 \mathrm{~K}$ are straight lines corresponding to

$$
{ }^{10} \log \sigma T=(4.6-1 / 2.3 k T) E_{\mathrm{a}}+1.33
$$

or

$$
{ }^{10} \log A=4.6 E_{\mathrm{a}}+1.33
$$

\section{Discussion}

In this section we discuss two possible explanations for the Meyer-Neldel behavior in the garnets. At first we consider the

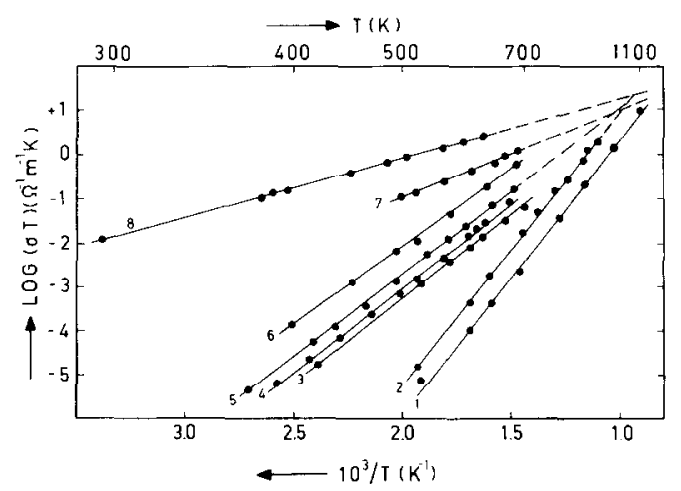

FIG. 2. Electrical conductivity vs reciprocal temperature for a single crystal $\mathrm{Ca}_{2} \mathrm{NaMg}_{2} \mathrm{~V}_{3} \mathrm{O}_{12-x}$. Numbers 1-8 indicate crystals with different values of $x$, due to a different reduction treatment at temperatures between 1100 and $1400 \mathrm{~K}$. Dashed parts of the lines are extrapolated values into the temperature region where reoxidation occurs.

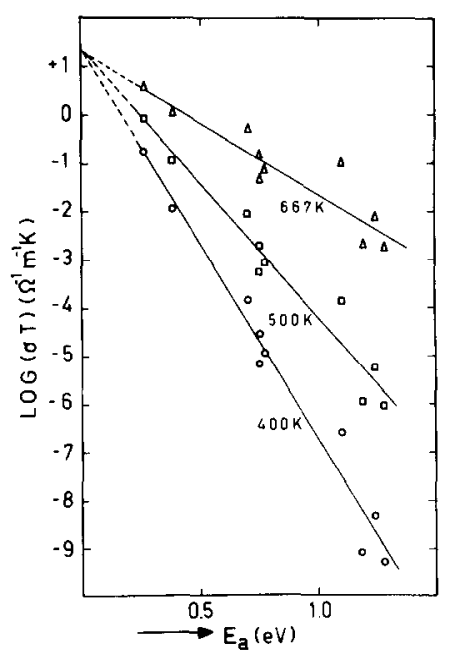

Fig. 3. ${ }^{10} \log \sigma T$ values from Fig. 2 at three different temperatures, plotted as a function of the activation energy. Straight lines demonstrate the Meyer-Neldel rule.

suggestion by Busch (5) that the factor $\alpha$ in Eq. (3) is a measure of the temperature $\theta$ where the defect concentration is frozen-in. However, the derivation given by Busch is not directly applicable in our case since Busch assumes that conduction takes place in a broad band, while charge transport in vanadium garnets takes place via adiabatic hopping of small polarons (27). Therefore, it is interesting to investigate whether the explanation of the Meyer-Neldel effect as given by Busch also holds for hopping conductors.

This is especially of interest since the assumption of Busch (5) that the compounds $\mathrm{UO}_{2}, \mathrm{Fe}_{2} \mathrm{O}_{3}, \mathrm{ZnO}, \mathrm{TiO}_{2}, \mathrm{BaTiO}_{3}$, and $\mathrm{SiC}$ are broad-band conductors is probably not justified. For instance, for $\mathrm{UO}_{2}(28), \mathrm{Fe}_{2} \mathrm{O}_{3}$ (21), and $\mathrm{BaTiO}_{3}(29)$ it has been assumed that the charge transport occurs via smallpolaron hopping. Notc also that Busch (5) and Weichman and Kuzel (6) write $E_{\mathrm{a}}=$ $\frac{1}{2} E_{\mathrm{d}}, E_{\mathrm{d}}$ being the donor ionization energy. This is only true when $N_{\mathrm{a}} \ll n \ll N_{\mathrm{d}}, N_{\mathrm{a}}$ being the acceptor concentration, $N_{\mathrm{d}}$ the donor concentration and $n$ the frec clectron concentration. This condition is only valid 
when the degree of compensation is very low. In many practical cases we have $n \ll$ $\left(N_{\mathrm{d}}-N_{\mathrm{a}}\right)$ and $n \ll N_{\mathrm{a}}$, in which case $E_{\mathrm{a}}=$ $E_{\mathrm{d}}$ instead of $E_{\mathrm{a}}=\frac{1}{2} E_{\mathrm{d}}$. However, this does not seriously alter the arguments.

At the temperatures where the measurements were performed, $T>\frac{1}{2} T_{\mathrm{D}}$, the mobility can be written as (21)

$$
\mu=\left(e a^{2} f / k T\right) \exp \left(-E_{\mathrm{a}} / k T\right) .
$$

Here $a$ is the intersite distance, $f$ the jump frequency, $E_{\mathrm{a}}$ the hopping energy, the other symbols having their usual meaning. This leads to

$$
\sigma T=\left(n e^{2} a^{2} f / k T\right) \exp \left(-E_{\mathrm{a}} / k T\right),
$$

when $n$ denotes the small-polaron concentration. At temperatures above the temperature $\theta$ where equilibrium with the surrounding atmosphere is maintained defects are formed by oxydation-reduction. For the case of the vanadium garnet

$$
2 \mathrm{~V}_{\mathrm{V}}+\mathrm{O}_{\mathrm{O}} \rightleftarrows \frac{1}{2} \mathrm{O}_{2}(\mathrm{~g})+\mathrm{V}_{\ddot{\mathrm{O}}}+2 \mathrm{~V}_{\mathrm{V}}^{\prime}
$$

Here the Kröger-Vink notation is used, $V_{V}$ denoting $\mathrm{V}^{5+}$ ions, $\mathrm{V}_{\mathrm{V}}^{\prime}$ denoting $\mathrm{V}^{4+}$ ions, and $V_{0} \ddot{o}$ denoting doubly ionized oxygen vacancies. The equilibrium constant for this reaction can be written as

$$
K=P_{\mathrm{O}}^{1 / 2}\left[\mathrm{~V}_{\mathrm{o}}\right]\left[\mathrm{V}_{\mathrm{V}}^{\prime}\right]^{2}=K_{0} \exp \left(-E_{\mathrm{v}}^{\prime} / k T\right) .
$$

Here square brackets indicate concentrations of the defects characterized by the enclosed symbols; $E_{\mathrm{v}}^{\prime}$ is the reaction energy of reaction (9). To maintain electroneutrality we have $\left[V_{\mathrm{V}}^{\prime}\right]=2\left[V_{0}\right]$. When the sample is cooled below a temperature $T=\theta$ the defect concentrations are frozen-in and the $\mathrm{V}^{4+}$ ion concentration is given by $\left[\mathrm{V}_{\mathrm{v}}^{\prime}\right]=$ const. $\exp \left(-E_{\mathrm{v}} / k\right)$, where $E_{\mathrm{v}}=\frac{1}{3} E_{\mathrm{v}}^{\prime}$. According to Eq. (8)

$$
\sigma T=C\left[\mathrm{~V}_{\mathrm{v}}^{\prime}\right] \exp \left(-E_{\mathrm{a}} / k T\right),
$$

where $C$ is a constant.

This equation can be brought into the
Meyer-Neldel form by assuming that the sum of the hopping energy and formation energy is constant: $U=E_{\mathrm{v}}+E_{\mathrm{a}}$. This leads to

$$
\sigma T=C \exp \left[-\left(U-E_{\mathrm{a}}\right) / k \theta\right] \exp \left(-E_{\mathrm{a}} / k T\right),
$$

where the constants of Eq. (3) are given by $\alpha=(k \theta)^{-1}$ and $\beta=\ln C-(U / k \theta)$.

Comparison with the experimental data given in Eq. (6) gives a value $\theta=1100 \mathrm{~K}$. Of course this value of $\theta$ can also be inferred directly from the extrapolated curves in Fig. 2. From experiments performed on a sensitive thermobalance and from the conductivity measurements we know that reoxidation of the samples starts around $1000 \mathrm{~K}$. Although there is no direct physical justification for the assumption that $U$ is a constant, the experiments therefore support the calculated $\theta$ value reasonably well.

In spite of this correspondence it is dangerous to conclude that both in broad-band and in narrow-band oxidic semiconductors the freezing-in of defects can generally explain the Meyer-Neldel behavior. Even for the vanadium garnet the actual situation is more complicated than suggested in Eq. (8). In Ref. (27) are presented results of conductivity measurements together with measurements of Seebeck coefficients of the garnets. A quantitative evaluation of these data indicates that interactions between charge carriers occur. Due to these interactions the activation energy $E_{\mathrm{a}}$ will decrease with increasing defect concentration. Since a detailed defect model is lacking, we have to make simplifying assumptions. Suppose that there are different pathways for the small-polarons, i.e., a polaron $i$ follows a path characterized by an effective hopping energy $E_{i}$. Assuming that the jump energies have a Gaussian distribution around the energy $E_{\mathrm{a}}, \sigma=\sum_{i} n_{i} e \mu_{i}$, with 


$$
\begin{gathered}
n_{i}=\left(N / w \pi^{1 / 2}\right) \exp \left(-\left(E_{i}-E_{\mathrm{a}}\right)^{2 / w^{2}}\right) \\
\mu_{i}=\left(e a^{2} f / k T\right) \exp \left(-E_{i} / k T\right)
\end{gathered}
$$

Replacing the summation over $i$ by an intcgration, we obtain

$$
\begin{array}{r}
\sigma T=\left(N e^{2} a^{2} f / k\right) \exp (w / 2 k T)^{2} \\
\exp \left(-E_{\mathrm{a}} / k T\right) .
\end{array}
$$

Both $w$ and $E_{\mathrm{a}}$ are functions of the defect concentration $x$ and therefore are interdependent: $w=f\left(E_{\mathrm{a}}\right)$. For instance, in the case of an ionic conductor with monopolemonopole interactions between the defects, it is shown in Ref. 26 that $w-C x^{1 / 2}$ and $E_{\text {a }}$ $=E_{\mathrm{a}, \mathrm{o}}-B x$, with $A$ and $B$ constants.

Elimination of $x$ gives

$$
\begin{aligned}
\ln \sigma T=\ln \left(N e^{2} a^{2} f / k\right) & +\frac{C^{2} E_{\mathrm{a}, \mathrm{o}}}{4 B k^{2} T^{2}} \\
- & \left(1+\frac{C^{2}}{4 B k T}\right) \frac{E_{\mathrm{a}}}{k T} .
\end{aligned}
$$

There will be a slight curvature in the $\ln \sigma T$ vs $T^{-1}$ plot, but over a limited temperature range this curvature is difficult to see.

An isothermal plot of $\ln \sigma T$ vs $E_{\mathrm{a}}$ will yield a straight line with slope

$$
\left(1+\frac{C^{2}}{4 B k T}\right) / k T
$$

Similar arguments can be used for broadband conductors when there is a Gaussian distribution of donor levels (or acceptor levels in case of $p$-type conductors) around the energy $E_{\mathrm{d}}$. The activation energy $E_{\mathrm{a}}$ in Eq. (12) is in this case equal to $E_{\mathrm{d}}$ or $\frac{1}{2} E_{\mathrm{d}}$.

In a slightly different approach Dosdale and Brook (17) show that under conditions where two exponential processes contribute and where one of the preexponential terms is constant, an approximately linear behavior is observed in a $\log A$ vs $E_{\text {a }}$ plot. These authors considered the case of diffusion (or ionic conduction) through polycrystalline materials where contributions are expected from both the bulk phase and from the grain-boundary region but, their arguments can be extended to our case of a single crystal with contributions from different polaron pathways.

\section{Conclusions}

Two explanations for the Meyer-Neldel rule in inorganic semiconductors have been discussed. It is shown that a theory based on freezing-in of defects, first given by Busch, can be extended to small-polaron hopping conductors. For this theory the constant $\alpha$ in the Meyer-Neldel relation (Eq. (3)) is directly related to the temperature where the concentration of the donor (or acceptor) defects is frozen in. The freezing-in temperature corresponds well with the expected value.

However, a Gaussian distribution of hopping energies or of donor or acceptor levels, together with specific defect interactions, can also lead to the Meyer-Neldel rule. Although such a distribution is expected when defect interactions are important, it is doubtful whether the interaction is of the right functional form in our case. Therefore the first explanation is preferable.

\section{References}

1. W. Meyer, Z. Phys. 85, 278 (1933).

2. W. Meyer, Z. Techn. Phys. 16, 355 (1935).

3. W. Meyer and H. Neldel, $Z$. Techn. Phys. 12, 588 (1937).

4. W. MeYER, Z. Elektrochem. 50, 274 (1944).

5. G. Busch, Z. Angew. Math. Phys. 1, 81 (1950).

6. F. L. Weichman and R. Kuzel, Canad. J. Phys. 48, 63 (1970).

7. F. Guimann and L. E. Lyons, "Organic Semiconductors," p. 428, Wiley, New York (1967).

8. B. Rosenberg, B. B. BhowmiK, H. C. Harder, and E. Posiow, J. Chem. Phys, 49, 4108 (1968).

9. G. G. Roberts and D. G. Thomas, J. Phys. C 7, 2312 (1974).

10. W. E. Speak, D. Allan, P. LeComber, and A. Ghaith, Philos. Mag. [Part] B 41, 419 (1980). 
11. D. E. Carlson and C. R. Wronski, in "Amorphous Semiconductors" (M. H. Brodsky, Ed.), Topics in Applied Physics: Vol. 36, Chap. 10, Springer-Verlag, New York (1979).

12. D. L. Staebler and C. R. Wronski, Appl. Phys. Lett. 31, 292 (1977).

13. D. L. Staebler and C. R. Wronski J. Appl. Phys. 51, 3262 (1980).

14. P. Irsigler, D. Wagner, AND D. J. Dunstan, $J$. Phys. C 16, 6605 (1983).

15. T. Dosdale And R. J. Brook, J. Mater. Sci. 13, 167 (1978).

16. T. Dosdale And R. J. Brook, Solid State Ionics 8, 297 (1983).

17. T. Dosdale and R. J. Brook, J. Amer. Ceram. Soc. 66, 392 (1983).

18. J. H. Gisolf, Ann. Phys. 1, 3 (1947).

19. A. Millem and E. Abrahams, Phys. Rev. 120, 745 (1960).
20. I. G. Austin and N. F. Mott, $A d v$. Phys. 18, 41 (1969).

21. A. J. Bosman and H. J. van Daal, Adv. Phys. 19, 1 (1970).

22. G. Kemeny and B. Rosenderg, J. Chem. Phys. 52, 4151 (1970).

23. G. Kemeny and B. Rosenberg, J. Chem. Phys. 53, 3549 (1970).

24. G. G. Roberts, J. Phys. C 4, 3167 (1971).

25. A. B. LidiaRD, in "Handbuch der Physik XX" (S. Flügge, Ed.), p. 246, Springer-Verlag, Berlin (1957).

26. K. E. D. Wapenaar, J. L. Van Koesveld, AND J. SChoonman, Solid State Ionics 2, 145 (1981).

27. G. Oversluizen and R. Metselaar, J. Phys. Chem. Solids, in press.

28. J. Devreese, R. De Coninck, and H. Pollak, Phys. Status Solidi 17, 825 (1966).

29. H. Ihrig, J. Phys. C 9, 3469 (1975). 